\title{
CRISIS AMBIENTAL Y CAMBIO CLIMÁTICO EN LA POLÍTICA GLOBAL: UN TEMA CRECIENTEMENTE COMPLEJO PARA AMÉRICA LATINA
}

\author{
Fernando Estenssoro Saavedra $\left({ }^{*}\right)$
}

\begin{abstract}
RESUMEN
El artículo analiza las proyecciones de la crisis ambiental global, particularmente su variable cambio climático, como posible fuente de tensiones y conflictos en el nuevo orden global emergente. Se plantea que de manera creciente la politología, los estudios internacionales y la geografía (entre otras disciplinas) consideran al factor ambiental y/o ecológico crecientemente determinante para la política mundial de la primera mitad del siglo XXI, al punto de plantear que la geopolítica de la globalización es la geopolítica del medio ambiente o la ambientalización de la geopolítica. En este sentido, el acento se pone en las miradas de potencias como los EE.UU. frente al tema, así como el papel que se le asigna a la América Latina, ejemplificando con el caso de la Amazonía.
\end{abstract}

Palabras clave:

Crisis ambiental - cambio climático - política mundial - geopolítica de la globalización - America Latina - Amazonía.

\begin{abstract}
The article discusses the projection of de global environmental crisis, particularly its variable climate change as a possible source of tension and conflict in the new emerging global order. It is argued that increasingly political science, international studies and geography (among other disciplines) consider the environmental factor and / or ecological increasingly crucial to world politics in the first half of this century,
\end{abstract}

$\left.{ }^{*}\right)$ Doctor en Estudios Americanos. Investigador del Instituto de Estudios Avanzados de la USACH. Especialista en Pensamiento Político, Época Moderna y Contemporánea.

Artículo recibido el 8 de mayo de 2010. Aceptado por el Comité Editorial el 23 de julio de 2010.

Correo electrónico: fernando.estenssoro@usach.cl 
to raise the point that the geopolitics of globalization is the geopolitical environment and greening of geopolitics. In this sense, the focus is on power looks like the U.S. front of the subject and the role that is assigned to Latin America, illustrating the case of the Amazon.

\section{Keywords:}

Environmental crisis - climate change - global politics - geopolitics of globalizationLatin america - Amazonia.

\section{INTRODUCCIÓN}

Es sabido que el Sistema Internacional, caracterizado por sus relaciones de poder asimétricas, siempre ha tenido apreciaciones diversas, confrontadas y difíciles de conciliar, respecto de la denominada crisis ambiental global, por lo menos desde que esta problemática se estrenó en agenda pública mundial hace ya más de 40 años. Estas distintas apreciaciones son las que finalmente explican las dificultades para avanzar en los acuerdos necesarios para mitigar una de sus importantes variables como es el problema del Cambio Climático, y que requiere de acuerdos vinculantes para todos los países de la comunidad internacional sobre reducción de gases efecto invernadero (en adelante GEI). Ningún actor del Sistema Internacional rechaza la urgente necesidad de alcanzar acuerdos multilaterales para solucionar este problema, producido por acción antropogénica, que está aumentando peligrosa y aceleradamente la temperatura media del planeta, por lo que también se le denomina "calentamiento global". Sin embargo, esfuerzos como los realizados en la Cumbre Mundial sobre Cambio Climático, efectuada entre el 7 y 18 de diciembre del año 2009 en Copenhague, no lograron responder a las expectativas de concordar un plan que pudiera sustituir y mejorar al protocolo de Kyoto a partir del 2012. ¿Por qué ocurrió este resultado? Es evidente que se trata de un tema de la agenda mundial contemporánea altamente relevante, sin embargo, su resolución es extraordinariamente compleja y no está claro cuán rápido se podrá avanzar en el futuro inmediato.

Al respecto, existe consenso entre los actores del sistema internacional de que si bien se trata de un fenómeno que incorpora diversas aristas (desde físicas hasta económicas y culturales) es, por sobre todo, un problema político en donde la variable espacial o geográfica adquiere gran relevancia. De aquí entonces, las enormes complejidades de las variables en juego, tanto para resolver la crisis ambiental en general, así como el Cambio Climático en particular, plantean desafíos y tensiones que se proyectan determinantes para el orden mundial emergente, al punto que de manera creciente distintos especialistas empiezan a plantear que la geopolítica en la era de la globalización, debe definirse como la "geopolítica de la sustentabilidad", o la "ambientalización de la geopolítica".

\section{LA BÚSQUEDA DE ACUERDOS PARA MITIGAR EL CAMBIO CLIMÁTICO}

Un primer elemento a considerar es el tema del Cambio Climático de raíz antropogénica, que remonta sus antecedentes a más de cien años en la agenda científica. 
Sin embargo, fue en la segunda mitad del siglo XX, y después que los estudios de Charles Keeling en 1957 demostraron que, efectivamente la concentración de $\mathrm{CO}_{2}$ en la atmósfera había aumentado desde la Revolución Industrial en adelante (Schoijet, 2008, p. 110), cuando éste se instala formalmente en la agenda política mundial, principalmente por los esfuerzos desplegados por la ONU para que se tomara conciencia del problema de la crisis ambiental global, y que tienen en la Conferencia sobre el Medio Ambiente Humano de Estocolmo en 1972, un hito fundamental. De aquí en adelante, la crisis ambiental será tratada como un macro problema real y donde cada una de sus variables (contaminación, agotamiento de los recursos naturales, pérdida de la biodiversidad, cambio climático, agujero de ozono, "explosión" demográfica) no sólo serán estudiadas en su especificidad, sino también en sus interrelaciones, buscando establecer acuerdos internacionales relativos a su superación.

En este sentido respecto de la variable del Cambio Climático o calentamiento global, destaca la Primera Conferencia Mundial sobre el Clima convocada por la ONU y realizada en 1979. Igualmente en 1983, se creó la Comisión Mundial del Medio Ambiente y del Desarrollo, que en su conocido informe Nuestro Futuro Común de 1987, volvió a insistir sobre la gravedad del tema del Cambio Climático y la urgencia de que éste fuera abordado por toda la comunidad internacional. En 1988 la Organización Meteorológica Mundial y el Programa de las Naciones Unidas para el Medio Ambiente crearon el Panel Intergubernamental sobre Cambio Climático más conocido por sus siglas en inglés IPCC (Intergovernmental Panel on Climate Change) destinado a realizar evaluaciones periódicas sobre este fenómeno y sus consecuencias. También se puede mencionar que en 1989, en París, se celebró la cumbre de los 7 países más industrializados (G7), donde el elemento convocante fue analizar "los estragos que se anticipan por efecto de los cambios climáticos globales" (Geisse, 1993, pp. 104-105). Todos estos eventos facilitaron el camino para que en la primera Cumbre de Medio Ambiente y Desarrollo Sostenible, efectuada en Río de Janeiro en 1992, se creara la Convención Marco sobre el Cambio Climático (en adelante la Convención). Posterior a la Cumbre de Río de Janeiro de 1992, cabe mencionar la realización de la "Cumbre de la Tierra +5 ", que tuvo lugar en un período extraordinario de sesiones de la Asamblea General de Naciones Unidas en Nueva York, entre el 23 y 27 de Junio de 1997, precisamente para analizar la ejecución de los acuerdo aprobados en la Cumbre de Río del 92. Lo interesante de esta Cumbre de la Tierra+5, fue el acuerdo de que los países debían "adoptar objetivos jurídicamente vinculantes para reducir la emisión de los gases de efecto invernadero, los cuales son causantes del Cambio Climático" (http://www.cinu.org.mx). Con este ambiente optimista, cinco meses más tarde, se llegó a una nueva cumbre de la Convención, efectuada en la ciudad japonesa de Kyoto en diciembre de 1997, cuyo objetivo fue cumplir con este mandato y obtener un acuerdo específico de disminución de la emisión de los GEI, por medio de la fijación de cuotas máximas de emisión por país. De esta forma, surgió el conocido Protocolo de Kyoto, donde los 30 países más industrializados del mundo fueron sus signatarios iniciales, comprometiéndose a reducir, como promedio, un 5,2\% de las emisiones de GEI entre los años 2008 y 2012, tomando como referencia los niveles de emisión del año 1990. Sin embargo este Protocolo no ha logrado sus objetivos. En 
primer lugar EE.UU., uno de sus firmantes iniciales, y que en su calidad de mayor emisor del mundo de GEI se había comprometido en Kyoto con una reducción de un 7\% (vale decir, por sobre el promedio de 5,2\%), nunca lo ratificó ya que lo estimó dañino para su crecimiento económico. Más aún, el expresidente G.W. Bush (hijo) retiró a EE.UU. del mismo en el 2001. En segundo lugar, este Protocolo recién entró en vigencia el 16 de febrero del año 2005, siete años después del encuentro en Kyoto, debido a que se había establecido que solo entraría a regir cuando lo ratificasen los países industrializados responsables de, al menos, un 55\% de las emisiones de $\mathrm{CO}_{2}$, quórum que sólo se logró tras arduas negociaciones, en noviembre de 2004 cuando Rusia lo ratificó. Y si bien es cierto, ya en 2006, 169 países lo habían ratificado, lo que implica $65 \%$ de las emisiones de $\mathrm{CO}_{2}$ del mundo, es claro que el Protocolo ya no logró las metas propuestas inicialmente, no sólo por la desafección de EE.UU., sino que además, se estima que no será posible disminuir las emisiones de GEI al nivel necesario para evitar que la temperatura media del planeta aumente por sobre $\operatorname{los} 2^{\circ} \mathrm{C}$ (por sobre esta cifra los científicos consideran que el problema se torna desastroso), si los países en vías de desarrollo no asumen también un compromiso obligatorio de reducción de sus emisiones. Recuérdese que el Protocolo de Kyoto estableció que la reducción de las emisiones debía empezar por los países desarrollados por ser los principales responsables de las mismas y planteó para los países en vías de desarrollo acciones voluntarias (Tickell, 2009, pp. 17-56; Schoijet, 2008, pp. 201-210).

En la medida que el plazo de expiración del Protocolo (2012), empezó a aproximarse, los países firmantes de la Convención del Cambio Climático, incluido EE.UU., se propusieron elaborar un plan para sustituirlo junto con "mejorarlo", en el sentido de hacerlo efectivo. Así, en diciembre del 2007, celebraron una nueva reunión en la Cumbre en Bali (Indonesia) y establecieron un documento $u$ "hoja de ruta de Bali", donde se dieron un plazo de dos años para a construir un nuevo acuerdo que permitiera responder a los objetivos iniciales de la Convención más allá del 2012. De esta forma se llegó a la Cumbre de Copenhague en diciembre de 2009, con la enorme expectativa de alcanzar un acuerdo de reducción de emisiones de los GEI que fuera vinculante para todos los países firmantes de la Convención, incluidos los países en vías de desarrollo. Objetivo que finalmente se frustró, prolongando así la incertidumbre (y las reuniones) respecto de la posibilidad de alcanzar un acuerdo eficaz para mitigar el calentamiento global.

Lo cierto es que el tema de avanzar de manera urgente en la reducción de la emisión de los GEI, es un proceso que está en plena evolución y todo indica que será cada vez más gravitante en la agenda política mundial. Para muchos científicos ya estamos enfrentando serias transformaciones del clima a raíz de la elevación de la temperatura media, lo que explicaría que tradicionales fenómenos climáticos, tales como sequías, huracanes, monzones, olas de calor y de frío (entre otros), sean cada vez más extremos y devastadores (IPCC, 2007, p. 53; Schoijet, pp. 130-186). Por lo tanto, ya no se trata de evitar que el "problema" suceda sino que mitigarlo antes de que se transforme en irreversible o que la denominada "retroalimentación positiva" del clima se transforme en inmanejable. 
Pero más allá de la evidencia científica, dado que este fenómeno se origina por causas sociales (antropogénicas), su solución es por definición un problema político. Particularmente, la discusión se focaliza en la emisión de dióxido de carbono (en adelante $\mathrm{CO}_{2}$ ), ya que dado su alto impacto en el efecto invernadero las discrepancias giran en torno a ¿cómo y dónde se disminuyen y/o mitigan sus emisiones y alta concentración en la atmósfera? En este sentido, el período de tiempo que se abre entre el avance de las consecuencias físicas del calentamiento global con el nivel y tipo de acuerdos para mitigarlo que se alcancen o no se alcancen, por parte del sistema internacional puede ser un periodo crecientemente conflictivo.

\section{EL FACTOR AMBIENTAL DE LA GEOPOLÍTICA DE LA GLOBALIZACIÓN}

En la medida que se ha desarrollado la conciencia de que nuestro mundo constituye un único y gran ecosistema, en donde una determinada acción o impacto de deterioro ambiental en un lugar del planeta puede repercutir en otro muy distantes y / o sobre el conjunto de la biósfera, también ha crecido la importancia del tema medioambiental en la agenda política mundial. En este sentido el tema relativo al aumento de la temperatura media del planeta, en la medida que se considera en extremo peligroso, ha venido cobrando creciente importancia en la geopolítica global ya que, como señaló el informe Stern, no sólo afectará gravemente a la economía mundial, sino que además implicará la propia existencia para muchas sociedades actuales, siendo las más pobres las que primero se verían afectadas.

$\mathrm{Al}$ respecto, no podemos olvidar que el ser humano, como toda especie viva "actúa primariamente en base a una lógica instintiva de mantenimiento y reproducción (supervivencia) como individuo y como especie. Y sólo cuando ha alcanzado la satisfacción de sus necesidades básicas, puede plantearse otro tipo de actuaciones o consumos" (Sánchez, pp. 42-43). Además, para asegurar su sobrevivencia, se comporta de manera gregaria (lo que define nuestro carácter social y político), así como necesita del espacio geográfico entendido como el medio que le aporta los recursos necesarios para su existencia.

De aquí entonces, si el equilibrio ecosistémico del espacio geográfico es clave para garantizar la vida y la seguridad de las sociedades y este equilibrio se ve amenazado por un fenómeno como el Cambio Climático, entonces las formas de uso y gestión de este espacio, desde la perspectiva ambiental, se transforma en un típico problema geográfico-político y estratégico. Al respecto, hoy en día son varios los politólogos y geógrafos que coinciden en este aspecto. Por ejemplo, Nogue y Vicente, plantean que "la escasez de los recursos naturales, los riesgos ecológicos, la relación entre crecimiento económico y degradación ambiental, el miedo a una crisis medioambiental global, la capacidad de movilización social de la ecología, el cuestionamiento por parte del medio ambiente de algunos aspectos de la soberanía de los estado-nación y el papel de los organismos internacionales", están llevando a la "ambientalización de la geopolítica" (Nogue y Vicente, 2001, p. 200). Beck, por su parte ha señalado que "con el discurso ecológico se experimenta todos los días el fin de la política exterior, el fin de los asuntos internos de otro país, o sea, el fin de los llamados Estados nacionales" 
(citado, en Caride y Meira, 2001, p. 48). Por cierto, no se trata de un cambio inmediato en el sistema internacional a raíz de la crisis ambiental, sino de un proceso que está en curso, pero en este proceso hacia un nuevo orden político global emergente, el factor ecológico puede ser un importante generador de conflictos por temas de soberanía. Como bien analiza Manuel Luis Rodríguez, desde el fin de la Guerra Fría, el orden mundial viene transitando de un esquema bipolar a otro multipolar, pero que éste aún no termina de consolidarse, y si bien el orden global multipolar será el escenario geoestratégico más probable de mediados del siglo XXI, este tránsito en que nos encontraríamos también implica "que nos acercamos a una prolongada etapa de rivalidad hegemónica entre potencias globales, mundiales y regionales por acceder a niveles mayores de la jerarquía de potencias en el sistema-planeta". Y en esta pugna,

la ecología y el medio ambiente serán factores de conflicto adicionales, tanto por la necesidad de los Estados de controlar el agua y preservar sus propios entornos naturales, como por el imperativo de cautelar su respectivo patrimonio ecológico territorial. Los riesgos de guerra ambiental, es decir de utilización de componentes del clima o del medio ambiente como instrumentos de destrucción masiva, podrían acentuarse en el futuro (Rodríguez, 2010).

Desde otra tradición epistemológica, Taylor y Flint, también concluyen que la problemática ecológica (o ambiental) lleva el conflicto geopolítico a un nivel cualitativamente distinto, sino definitivo. Para ellos, hoy en día existe la lógica de la ecología global que nos lleva a representarnos al planeta como el "hogar de la humanidad", y nos hace tomar conciencia de que estamos destruyendo este hogar "por nuestra cuenta y riesgo", y por lo tanto se enfatizará la demanda de su conservación dado que, finalmente, se trata de nuestra propia conservación. Sin embargo, también existen otras lógicas globales, como la lógica de la globalización financiera, que concibe al mundo "como un lugar, una plataforma abstracta para la acción en la que operar, por ejemplo el espacio financiero de veinticuatro horas al día de las ciudades mundiales". Así, mientras en una lógica nos preguntamos si el espacio geográfico es "sostenible", en la otra nos preguntamos si es "eficiente". De esta forma, en la geopolítica del mundo globalizado actual coexisten variables muy distintas como la preocupación por "la conservación de la biodiversidad de las selvas tropicales" junto con "el mantenimiento de la competitividad de Londres" dentro del concierto de las ciudades mundiales. Esta situación puede ser altamente confrontacional, si se considera que el modelo de vida hegemónico impuesto por los EE.UU., se caracteriza, esquemáticamente, por el elevado nivel de consumo. Sin embargo, dada la gran cantidad de habitantes del planeta y sus proyecciones de crecimiento, no es posible a la Tierra sostener ese estándar y estilo de vida para todos (o sea, si el consumo humano es llevado a unidades de energía, no hay suficiente energía en el planeta para que todos sus habitantes alcancen el nivel de consumo de un ciudadano estadounidense promedio). Por lo tanto, concluyen estos autores, lo que mucha gente plantea es "que las tendencias actuales son insostenibles desde el punto de vista ecológico". De aquí entonces, afirman que la "la crisis ecológica global es la tensión definitiva entre el lugar y el espacio, entre hacer de la Tierra un lugar habitable y explotarla como espacio de recursos" (Taylor y Flint, 2002, p. 409). 


\section{CAMBIO CLIMÁTICO Y PROBLEMA ENERGÉTICO, UN SOLO FENÓMENO CON DOS CARAS}

Otra característica de la geopolítica de la globalización, es que el tema del Cambio Climático está indisolublemente ligado a un tema estratégico mucho más tradicional como es el referido al acceso a las fuentes de energía. En otras palabras, en el actual contexto de la discusión mundial sobre el Cambio Climático, la variable ambiental y la variable energética se transforman en un sólo fenómeno cuyo derrotero puede ser determinante para la historia del presente siglo. Esto es así porque nuestra llamada Civilización Industrial, depende de los combustibles fósiles (carbón, gas y petróleo), particularmente del petróleo, como fuente de energía primaria. Y, como es sabido, los combustibles fósiles son los grandes emisores de dióxido de carbono $\left(\mathrm{CO}_{2}\right)$, que a su vez es el más importante de los gases efecto invernadero (GEI) de raíz antropogénica, con más del $76 \%$ del total de éstos.

$\mathrm{Al}$ respecto, cabe recordar que a la Revolución Industrial se la culpa de transformar el calentamiento global en un problema porque estimuló la utilización masiva de los combustibles fósiles como fuente de energía, "inicialmente el carbón, el petróleo a partir de 1859, y posteriormente el gas natural" (Schoijet, 2008, p. 23). Además, con la aparición de las máquinas eléctricas y de las redes eléctricas desde 1880 en adelante y el auge del automóvil desde 1890, durante todo el siglo XX aumentó exponencialmente el consumo de éstos, particularmente el petróleo, que resultó ser una extraordinaria y versátil fuente energética. Así, tras el término de la Segunda Guerra Mundial, el crudo pasó a sustentar el espectacular crecimiento económico-industrial del Primer Mundo, dado que se trataba, entre otras razones, de un recurso "abundante" y "barato", por lo menos hasta la guerra árabe-israelí de 1973. Y si bien es cierto, en nuestros días existe una discusión respecto de si se habría alcanzado el peak de su producción y / o se estaría próximo a alcanzarlo (lo que llevaría a su progresivo encarecimiento), éste se ha convertido en el componentes más importante de la matriz energética mundial, con el $40 \%$ de toda la energía empleada. Además su versatilidad lo ha transformado en una sustancia en extremo útil para el modo de vida contemporáneo, al punto que es común escuchar que vivimos en la era del petróleo o civilización del petróleo (Palazuelos, 2008; Rifkin, 1999).

Ahora, si consideramos que el petróleo y el gas natural representan cerca del 63\% de la matriz energética primaria mundial, y que el carbón representa algo más del 25\% de la misma, tenemos entonces que en la primera década del presente siglo XXI, el $85 \%$ de las necesidades energéticas del mundo son cubiertas por combustibles fósiles, principales emisores de $\mathrm{CO}_{2}$. Y, para hacer aún más complejo este problema, todas las proyecciones señalan que, salvo algún fenómeno extraordinario, la demanda de energía seguirá aumentando producto de una economía mundial impelida por alcanzar cada vez más altos estándares de vida para una población mundial también en aumento, en donde una gran mayoría busca salir de la pobreza y el subdesarrollo, y una minoría busca mantener y/o aumentar su alto estándar ya alcanzado.

El gran problema, es que durante las próximas décadas la matriz energética seguirá 
dependiendo de los combustibles fósiles, particularmente del petróleo. Como señala el Centro de Investigaciones Energéticas, Medioambientales y Tecnológicas de España (CIEMAT), en un estudio sobre las proyecciones realizadas por la Energy Information Administration de los EE.UU., el consumo de energía a nivel mundial se incrementará en un 57\% entre 2004 y 2030, y los combustibles fósiles, seguirán siendo los más utilizados, por su importancia en el transporte y en el sector industrial. Específicamente el consumo de petróleo aumentaría de 83 millones de barriles día en el 2004 a 97 millones de barriles día en el 2015 y a 118 millones en 2030 (Ciemat, 2006). Otros autores citan informes de la CIA donde se señala que para el 2020 se espera que el consumo energético mundial aumente considerablemente, ya que si en el período 1980-2000 éste aumentó en un 34\%, para el período 2000-2020 aumentaría en un 50\%, y la base de este aumento seguirá siendo el petróleo, en cambio las energías renovables "no superarían el 8 por ciento del abastecimiento energético" (Sohr, 2009, p. 23).

La importancia estratégica del petróleo es tan gravitante, que el presidente estadounidense, Barack Obama en marzo del 2010, autorizó la reapertura de las exploraciones petrolíferas off shore en la costa atlántica y el Golfo de México, que estaban suspendidas por casi 20 años, así como señaló que mantendrá las exploraciones petroleras en el territorio de Alaska iniciadas por la administración anterior del presidente Bush (La Tercera, 1 de abril de 2010, p. 11). Este anunció no fue menor dado que el discurso que caracterizó a Obama durante la campaña electoral, tenía un fuerte acento ambientalista y centrado en la promoción de las energías renovables y "limpias" para enfrentar el Cambio Climático. Sin embargo, cuando se asocia el tema del calentamiento global al problema de la seguridad energética de los EE.UU., queda claro cual es la coherencia lógica que impulsa al presidente estadounidense. Recuérdese que ya el 26 de enero del 2009, impartió medidas que asociaban el combate al Cambio Climático con la independencia energética del país, cuando señaló que los fabricantes de automóviles deberían producir vehículos más eficientes para el 2011 y esperaba que para el 2020 el rendimiento fuera de 35 millas por galón. Entonces planteó que los "Estados Unidos no será rehén de recursos cada vez más limitados, de regímenes hostiles y del calentamiento del planeta. No nos quedaremos de brazos cruzados sólo porque actuar es difícil (...) ningún asunto es tan fundamental como el de la energía" (El Mercurio, 27 de enero de 2009). Por lo tanto, si bien Obama estaría decidido a modificar la actual matriz energética a fin de disminuir las emisiones de $\mathrm{CO}_{2}$, sabe que pasarán muchos años antes de que los EE.UU. deje de ser una sociedad dependiente del petróleo. Luego, durante todo el periodo de transición que iría desde la actual matriz energética "sucia", hasta alcanzar una nueva era de matriz energética "limpia" (y que puede durar décadas), será clave el abastecimiento de crudo para funcionamiento del país, y en este sentido, dado que las principales fuentes de abastecimiento están en zonas poco estables y/o inseguras desde la perspectiva de la superpotencia mundial (Medio Oriente, Venezuela, Cáucaso, otras), los EE.UU. deben aumentar su propia producción.

En síntesis, pese a los avances y esfuerzos que se han realizado por diversificar la matriz energética a nivel mundial, esta sigue dependiente de los combustibles fósiles. Situación que se proyecta similar, por lo menos hasta mediados del 
presente siglo y probablemente más allá. De aquí entonces, ya se prefiera hablar de Civilización Industrial o Civilización del Petróleo, en estos tiempos de globalización y de calentamiento global, ambas variables: energía y medio ambiente, son un solo fenómeno geopolítico. Por estas razones, John P. Holdren, Director The Woods Hole Research Center y Presidente de la American Association for the Advancement of Science, ha señalado que la "energía es el centro del problema ambiental, el ambiente está en el centro del problema energético, y resolver el dilema energía-economía-ambiente es el núcleo del problema del bienestar sustentable tanto para las naciones industrializadas como en desarrollo" (citado en Zanelli, 2009). Opinión similar a la del politólogo canadiense Homer-Dixón, quien también ha señalado que la crisis del Cambio Climático y la dependencia de nuestra civilización de los combustibles fósiles, son un sólo problema que él sintetiza como el "problema del carbono" (CO2). Dependemos sobre todo del petróleo, pero también del gas y carbón, como fuente energética y a la vez estos son grandes emisores de $\mathrm{CO}_{2}$ a la atmósfera, por lo tanto la solución del calentamiento global y la solución de contar con fuentes energéticas "limpias" de CO2 son inseparables (Homer-Dixon, 2009).

\section{LA DIMENSIÓN ESTRATÉGICA DEL CAMBIO CLIMÁTICO PARA LAS POTENCIAS PRIMERMUNDISTAS}

Lo anterior quiere decir que la solución de la ecuación compuesta por las variables: disponibilidad de recursos energéticos y crisis ambiental, se transforma en determinante para el devenir de los asuntos políticos mundiales, regionales y locales, dado que su discusión nos retrotrae al tema de la supervivencia misma y explica porqué el tema se viene transformando en un tópico político estratégico prioritario.

Por cierto, existe un amplio consenso de que la crisis ambiental en general, y el calentamiento global en particular, son problemas globales. Sin embargo, son conocidas las diferentes perspectivas entre países desarrollados y países en vías de desarrollo, respecto dónde y cómo se ponen los énfasis de la solución para superar tan complejo problema. En nuestro desigual mundo, medidas que resultan evidentes y "naturales" para sociedades que viven con un PIB promedio de 40 mil dólares pér cápita, pueden no ser tan evidentes para aquellas que viven con 10.000 dólares pér capita y menos. Por cierto, este es un tema que viene siendo tratado largamente por la ONU, buscando visiones compartidas y políticas multilaterales que permitan alejar la amenaza de conflictos violentos derivados de la crisis ambiental y la desigual distribución de la riqueza a nivel mundial. Sin embargo, para conocer las complejidades del orden global en gestación es conveniente tener presente algunas de las principales ideas que vienen articulando la visión geopolítica y estratégica del Primer Mundo a raíz de la crisis ambiental y el cambio climático, y que manifiestan grados de tensión entre el Norte y el Sur.

Por ejemplo, si tomamos el caso de la principal superpotencia mundial, los EE.UU., vemos que tempranamente aparece la preocupación respecto de las posibles implicancias que la crisis ambiental y/o alguna de sus variables específicas pudieran significar a su seguridad nacional, así como para la estabilidad política mundial. De 
hecho a mediados de los años setenta del siglo pasado, el entonces presidente Jimmy Carter, solicitó al Departamento de Estado un estudio demográfico y medioambiental sobre cómo sería el estado del mundo a inicios del siglo XXI y qué consecuencias podría traer al país. De esta forma surgió el Informe Global 2000 para el Presidente, en donde se señaló la posibilidad de serias crisis en la disponibilidad de recursos naturales, en el medio ambiente y en la estabilidad política y económica debido al acelerado crecimiento demográfico del mundo. Años más tarde, en 1991, el medio ambiente fue incluido por el presidente George Bush (padre) como variable de análisis de la Estrategia de Seguridad Nacional del Departamento de Defensa de los Estados Unidos (Nogué y Vicente, p. 189). Posteriormente, bajo la presidencia de Bill Clinton, en marzo de 1996, Anthony Lake, consejero presidencial para los asuntos de seguridad nacional, en un discurso pronunciado en la George Washington University, señalaba que entre las amenazas directas a la seguridad del país o los "peligros hostiles", se encontraba, junto al terrorismo y otros, la "depredación ambiental" (Joxe, 2003, p. 189). De la misma forma el entonces Secretario de Estado, Warren Christopher, señalaba en la University of Stanford, que la administración Clinton estaba absolutamente convencida que su capacidad de llevar adelante los intereses mundiales estaba ligada a la ordenación de los recursos naturales de la Tierra. Por lo tanto, estaban "decididos a situar las cuestiones medioambientales en el lugar que le corresponde, junto con otras prioridades de la política exterior de Estados Unidos", que finalmente se orientan a asegurar el bienestar y la supervivencia de su población (Christopher, 1996). De manera más reciente, en el año 2004, el Pentágono planteó al presidente George Bush (hijo) que el Cambio Climático implicaba una amenaza aún mayor para la seguridad de los Estados Unidos, que el fenómeno del terrorismo fundamentalista que había derribado las Torres Gemelas de Nueva York, el 11 de septiembre del 2001.

En un informe secreto, sobre la base de un estudio que habría sido elaborado por Peter Schwarz y Doug Randall, pertenecientes a la organización empresarial Global Businnes Network, se advertía (entre otros aspectos) que a raíz del calentamiento global, en los próximos 20 años las mega-sequías y el hambre provocarían revueltas masivas y migraciones catastróficas, e incluso guerras nucleares, en todo el mundo y por tanto EE.UU. debía construir fortalezas defensivas tendiente a proteger al país de los inmigrantes potenciales por el cambio climático (Towsend y Harris, 2004; Schoijet, pp. 229-230). En noviembre del 2007, un grupo de investigadores de dos centros de estudios internacionales y políticos estadounidenses, el Center for Strategic \& International Studies y el Center for a New American Security, publicaron The Age of Consequences:Policy and National Security. Implications of global climate change, estudio donde se vuelve a insistir que a raíz del calentamiento global, la seguridad de los EE.UU., se vería crecientemente amenazada por fenómenos tales como: la emergencia de conflictos bélicos por recursos de agua dulce, masivas migraciones de personas desplazadas, aumento de la construcción de reactores nucleares para abastecimiento energético, enfermedades infecciosas cada vez más frecuentes y mortales, entre otros problemas. Situación que, además, provocaría el realineamiento de poderes en el orden internacional, donde Rusia volvería a jugar un papel protagónico debido a sus ricas fuentes de gas natural (Campbell, et. al, 2007). Igualmente el 2008, Thomas Fingar, Presidente del National Intelligence Council (NIC) daba a conocer el estudio prospectivo 
realizado por las 16 agencias de inteligencia de EE.UU., Trends 2025: A Transformed World. En éste, nuevamente se insiste en que el calentamiento global es una amenaza real para la seguridad del país, ya que agravará la pobreza mundial, desestabilizará a los Estados más frágiles del planeta (lo que estimularía las migraciones de los más pobres al Primer Mundo), así como favorecería a las tendencias terroristas. Además, tempestades cada vez más agudas podrían afectar a las plantas nucleares en territorio estadounidense y a las plataformas petroleras off shore, entre otras consecuencias (NIC, 2008). De manera aún más reciente, en febrero del 2010, se dio a conocer el informe The Joint Operating Environment 2010, realizado por el Joint Forces Command (JFC), del Departamento de Defensa de los EE.UU. En él, se reiteran las principales tendencias que podrían amenazar la seguridad de la superpotencia y de todo el Sistema Internacional en la primera mitad del siglo XXI. Al igual que en los informes anteriores, estas se inician con los problemas asociados al aumento de la población mundial, que para el 2030 podría llegar a los 8 mil millones de personas (el 95\% de este aumento ocurriría en los países en vías desarrollo). También se resalta la escasez de agua dulce, precisamente a raíz del aumento de la población, así como de la contaminación creciente de los cursos de agua, fenómeno que ocurriría principalmente en los países en vías de desarrollo. También se señala que el aumento de la población implicará el aumento de la demanda de energía y, salvo la ocurrencia de cambios dramáticos en la tecnología junto con una inserción masiva de capital en energías renovables y una drástica alteración de las actitudes políticas hacia la energía nuclear, este aumento de energía será de la mano del aumento del consumo combustibles fósiles. En este mismo sentido, también señala que el Cambio Climático, se ha convertido en una preocupación creciente para las FF.AA. del país y se visualiza como una de las 10 tendencias que tendrá un mayor impacto para el desenvolvimiento de las fuerzas conjuntas. Por ejemplo, el deshielo del Ártico a raíz del calentamiento global aumentaría la tensión estratégica de esa zona del planeta, dado que se transformaría en ruta privilegiada de navegación entre el Pacífico y el Atlántico, además ya existirían preocupantes indicios de reclamación de soberanía de su lecho marino, como la instalación de una bandera rusa en el fondo del mar correspondiente al polo ártico en 2007 (JFC, 2010, pp. 12-37). A lo que debe agregar que el Instituto de Investigaciones Geológicas de EE.UU. (USGS, según sus siglas en inglés) dio a conocer en julio del 2008, un estudio donde las reservas de petróleo que estarían bajo los hielos árticos (que podrían ser explotadas tras el derretimiento de los hielos o la implementación de nuevas tecnologías), llegarían a uno 90.000 millones de barriles, además de otros 48 billones de metros cúbicos de gas natural. Esta situación, unida al hecho que cinco países reclaman soberanía sobre este espacio geográfico (Canadá, EE.UU., Rusia, Dinamarca y Noruega), lo proyecta como una zona de futuros y agudos conflictos (Vera y Luco, El Mercurio, 2008, B, p. 26).

De aquí entonces, se entiende por qué Joseph Nye, señala que la acción de los Estados Unidos por influir sobre la política de otros estados y organismos no estadounidenses obedece a la necesidad de proteger la seguridad de sus ciudadanos que se ven amenazados por fenómenos que ocurren más allá de sus fronteras. Y, por cierto, entre las nuevas amenazas, ahora se encuentran las relacionadas al deterioro ecológico del planeta, como es el caso del Cambio Climático y la conservación de especies en peligro, entre otros aspectos (Nye, 2003, pp. 197-223). 
Pero esta perspectiva del problema no sólo es propia de los EE.UU., sino que es compartida por otros influyentes países del Primer Mundo. Por ejemplo, Olabe y Mikel, señalan que el impacto de la crisis climática sobre la seguridad mundial fue discutido en el 2007 por el Consejo de Seguridad de la ONU a petición de Reino Unido y también ha sido tratado como "problema de seguridad" en la Cumbre de Primavera del 2008 de la Unión Europea (Olabe y Mikel, 2008, p. 175). Igualmente, desde la academia canadiense Homer-Dixon, se ha caracterizado por señalar que el "estrés energético" a raíz de la crisis de producción del petróleo, unido al "estrés ambiental", con problemas como la deforestación, la falta de agua y crecimiento demográfico; además del "estrés del Cambio Climático", entre otras tensiones globales, se están transformando en una amenaza catastrófica para el orden mundial (Homer-Dixon, 2006).

\section{UN TEMA DE INTERÉS PARA AMÉRICA LATINA: LOS ECOSISTEMAS COMO UN NUEVO TIPO DE RECURSOS ESTRATÉGICO}

El tema de la crisis ambiental ha dejado de manifiesto la necesidad de protección de la naturaleza en su conjunto. Como bien señala el director de Estudios Prospectivos de UNESCO, Jerome Binde, la principal enseñanza que dejó la Segunda Cumbre de Desarrollo Sostenible y Medio Ambiente, el 2002 en Johanesburgo, es que "no se pueden oponer, desarrollo sostenible y desarrollo a secas, lucha contra la pobreza y conservación de los ecosistemas". Por el contrario, es necesario "luchar en todos los frentes a la vez: a partir de ahora, nuestro enfoque debe ser global, lo mismo que nuestra conciencia" (Binde, 2008, p. 104). Sin embargo, dependiendo del problema específico que se analice, existen indudablemente ecosistemas que son más importantes que otros para el sostén de la "salud ambiental" global, y frente a la conservación de estos ecosistemas considerados "vitales", la idea de luchar contra la pobreza y al mismo tiempo conservarlos, no se ha traducido hasta el momento en un política realmente efectiva a nivel mundial, sino que es más bien una declaración de buenas intenciones que busca bajarle el perfil al creciente grado de tensión que existe en este tema entre los países más y menos desarrollados.

Esta tensión no es menor para América Latina, dado que tanto en la variable protección de la biodiversidad del planeta, como en la de mantener los ecosistemas que son fundamentales para mitigar el Cambio Climático (porque absorben de manera natural el dióxido de carbono), el espacio biogeográfico de esta parte del mundo viene adquiriendo una importancia en extremo gravitante, particularmente porque aun tiene ecosistemas poco intervenidos por el ser humano y la presión primer-mundista para que se mantengan así está aumentando. Sin embargo, como es sabido los países en vías de desarrollo como los latinoamericanos y caribeños, aún dependen de la explotación primaria de sus recursos naturales como sostén para su desarrollo y crecimiento económico, por lo tanto la ecuación no intervención industrial de los ecosistemas y crecimiento y desarrollo económico, es un tema de difícil resolución para esa parte del mundo.

\section{El caso de la Amazonía}

Un ejemplo clarificador es la discusión que ocurre con relación al destino del ecosistema 
amazónico o Amazonía, ya que bajo la lógica de considerar que la humanidad se encuentra en una época histórica determinada por la crisis ambiental global, se asigna a esta porción del espacio terrestre un valor significativo tanto en lo que respecta a la conservación de la biodiversidad como a la mitigación del calentamiento global.

Por ejemplo, en el tema de conservar la biodiversidad existen dos planteamientos principales: proteger las especies y las poblaciones individuales o proteger los hábitat en los que viven. Y en este sentido, según los especialistas, la más importante de las dos es la conservación de los ecosistemas enteros (hábitat natural), asegurando su funcionalidad. Ahora si consideramos que la destrucción de los ecosistemas es la mayor amenaza para biodiversidad y esta destrucción ha ocurrido fundamentalmente por la intervención del ser humano, resulta entonces que la conservación "prístina" de los ecosistemas no intervenidos o muy poco intervenidos es una exigencia creciente. Así, se señala que sólo quedarían sin transformar por el hombre el 51,9\% de las tierras emergidas (aproximadamente 90 millones de $\mathrm{km}^{2}$ ), pero si se excluyen las áreas desérticas, rocosas y heladas, las zonas no transformadas o con sus ecosistemas poco intervenidos son sólo el $27 \%$ de las tierras emergidas. En este mismo sentido, América del Sur tiene el 62,5\% de su territorio poco intervenido, de manera principal el ecosistema amazónico; e igualmente Oceanía tiene el 62,3\% de su territorio bien conservado, que corresponden fundamentalmente a los desiertos de Australia (MappingInteractivo, 2001; Hannah, Carr, Lankerani, 1995, pp. 128-155).

Ahora respecto de la variable del Cambio Climático, es conocida la preocupación por la conservación y preservación de principales sumideros naturales de $\mathrm{CO}_{2}$ como son las masas boscosas y los océanos del planeta. Para nuestro análisis, el caso de las masas boscosas es relevante dado que todas ellas se localizan en algún lugar del espacio terrestre que pertenece o es reclamado como propio por algún Estado nacional, a diferencia de los océanos cuya mayor superficie se considera "alta mar".

Si consideramos que la Amazonía, con sus 6,5 millones de $\mathrm{Km}^{2}$, es identificada como una de las principales masas boscosas del globo (selva húmeda tropical), dado que contiene la mayor biodiversidad del planeta y además eso uno de los sumideros naturales de $\mathrm{CO}_{2}$ más importantes que existen (de aquí su mención como "pulmón del planeta"), queda claro que su proyección geopolítica se ha complejizado aun más, particularmente para el Brasil, que tiene la mayor proporción del ecosistema amazónico $(66 \%)$. De hecho Schoijet señala que si bien en el siglo XIX y primera parte del XX, cuando la ecología estaba en pañales, nadie cuestionaba "el derecho de cualquier país a utilizar sus recursos naturales, incluyendo sus tierras cultivables", pese a que se originaron enormes desastres ambientales (como el caso de la colonización del Dust Bowl), la "deforestación de la Amazonía y el cambio climático se produce en una situación históricamente nueva, en que la ciencia se ha desarrollado lo suficiente como para prever que la combinación de ambos procesos lleva a un desastre probablemente mucho mayor (...) que seguramente afectará a muchos países". De aquí entonces, resulta "imprescindible limitar la soberanía del Brasil sobre la Amazonía, condicionándola a que su gobierno detenga la deforestación" (Schoijet, p. 151). 
Por lo tanto, si la geopolítica tradicional visualizaba a la Amazonía como un inmenso territorio riquísimo en recursos naturales y poco poblado, ahora, frente a la crisis ambiental, ha adquirido nuevo valor estratégico, en su calidad de "pulmón natural del planeta" y reservorio de biodiversidad. Así, su valor ha aumentado y con ello el interés de distintos poderes económicos y políticos internacionales y transnacionales por su posesión y gestión (Fregapani, 2000).

Como es sabido, el interés de las grandes potencias Europeas y de los Estados Unidos por la Amazonía se remonta al siglo XIX y primera parte del XX, por cierto bajo los argumentos propios de los momentos histórico-político específicos de entonces. Y si bien, ya el Presidente de EE.UU., Woodrow Wilson, planteó tempranamente su internacionalización como bien común y patrimonio de la humanidad dada su característica de "pulmón del planeta", será en la segunda mitad del siglo XX, con el tema de la crisis ambiental global, cuando la Amazonía recobra un extraordinario interés del ahora llamado Primer Mundo, alarmado por el proceso de deforestación y "devastación" que sufriría producto de la explotación económica que hacen de ella (o permiten que se haga) los países que contienen su ecosistema.

Por cierto, con el avance que ha tenido el debate mundial sobre la crisis ambiental, hoy día nadie pone en duda el papel fundamental que le cabe a un ecosistema como el amazónico para sostener el equilibrio ecológico del planeta, pero resultará inaceptable para los países latinoamericanos en general y para el Brasil en particular, que desde el Norte se les dicte cómo deben actuar y se les intente fijar "el papel de guardianes del equilibrio ecológico mundial, sujetos a reglas dictadas por centros foráneos", ya que, entre otras cosas, "se estaría subsidiando la modalidad de desarrollo, altamente deteriorante del medio ambiente, que continúan privilegiando los países del mundo industrializado" (Comisión de Desarrollo, 1991, p. 18). Así cuando el Presidente de Brasil, Luiz Inácio Lula da Silva, señala que "ningún país tiene un interés mayor en reducir el impacto del calentamiento global que Brasil. Es por esto que estamos en la primera fila de las iniciativas para encontrar soluciones que preserven nuestro futuro común" (Sauven, La Nación, Santiago, 10 de junio de 2009, p. 19); su pensamiento debe ser complementado con las declaraciones realizadas por su Ministro de Defensa, Nelson Jobim, que en un discurso ante el Jefe del Estado Mayor de Estados Unidos, Mike Mullen, planteó: "Tenemos exactamente la intención de ser una potencia, participando en América Latina y recordando siempre que la Amazonía brasileña es cuidada por los brasileños en favor de Brasil y del mundo. O sea, no hay tutela posible sobre la Amazonía que no sea la brasileña" (La Nación, Santiago, 3 de marzo de 2009).

En este sentido, los países latinoamericanos (y los en vías de desarrollo en general) han sostenido permanentemente que junto con el respeto de su soberanía por parte del Primer Mundo, no se puede tratar el tema de la crisis ambiental de manera unilateral, o sólo enfatizando lo que preocupa a los países más ricos. En este sentido, el planteamiento permanente de los países en vías de desarrollo, es que la causa de la crisis ambiental radica en la histórica asimetría en el desarrollo humano y económico que caracteriza a un mundo donde el llamado Primer Mundo, logró su alto estándar de vida sobre la base de un sistema económico altamente contaminante y depredador 
de recursos naturales que "encadenó" al Tercer Mundo a la función de proveedores de materias primas y fuerza de trabajo. Por lo tanto, la solución a la misma, no puede ser sobre la base de clausurar el derecho al desarrollo y crecimiento de los más pobres.

Precisamente, respecto de la deforestación de la Amazonía, se señala que esta obedece, por sobre todo, a la presión por ganar espacios para realizar una agricultura destinada a commodities como la soja, o el café, donde los principales mercados consumidores y demandantes de los mismos están en el Primer Mundo (Procopio, 2009). Por su parte, el Presidente del Ecuador, Rafael Correa, de acuerdo con la idea-fuerza latinoamericana de que "no hay peor enemigo para el medio ambiente que la pobreza", propuso a la Convención de Países para el Cambio Climático, reunida en Polonia el 2008, la revolucionaria alternativa que para proteger el ecosistema amazónico del Parque Natural Yasuní, donde se ubican las mayores reservas de petróleo del país, Ecuador cancelaría los planes para su explotación petrolera y velaría por conservación del ecosistema, siempre y cuando se le pagara a su país el ingreso equivalente a los 920 mil millones de barriles de crudo que se calcula existen y que dejaría de obtener por su no explotación (El Mercurio, 11 de diciembre de 2008, A5).

En síntesis, lo que se señala desde América Latina es que la principal lógica que impera y ordena el sistema internacional, es la de un intercambio económico desigual que ha existido entre el centro y la periferia desde la era moderna en adelante y que no ha cambiado en la actual época de globalización. Así, lo que ha ocurrido frente a fenómenos como la crisis ambiental, es que esta lógica de poder e intercambio desigual no ha cambiado sino que se ha hecho más compleja. Vale decir, si antes de que existiera conciencia de la crisis ambiental, el papel asignado a la periferia por el centro era fundamentalmente el de proveedor de materias primas, ahora también se le ha asignado el papel de sostenedor y conservador de ecosistemas que se consideran vitales para el funcionamiento del planeta y que, precisamente, se ubican el en Sur del mundo. Al respecto, resulta interesante el planteamiento de Leff, Argueta, Boege y Porto Goncalves, cuando señalan:

... las políticas recientes en torno a la biodiversidad no responden tan sólo a una preocupación por la pérdida de especies biológicas y por su importante papel en el equilibrio ecológico del planeta. La biodiversidad se ha revelado como un enorme banco de recursos genéticos que son la materia prima de los grandes consorcios de las industrias farmacéuticas y de alimentos, cuyo valor económico supera ya el de los consorcios petroleros (...) La geopolítica de la biodiversidad y del desarrollo sustentable no sólo prolonga e intensifica los anteriores procesos de apropiación destructiva de los recursos naturales, sino que cambia las formas de intervención y apropiación de la naturaleza cuestionando la sustentabilidad posible de la racionalidad económica (...) Así, ante el calentamiento global del planeta (...) la geopolítica del desarrollo sostenible mira con optimismo la solución de las contradicciones entre economía y ecología al proponer la reconversión de la biodiversidad en colectores de gases de efecto invernadero, con lo cual se exculpa a los países industrializados de sus excedentes de sus cuotas de emisiones, mientras se induce una reconversión ecológica de los países del tercer mundo (...) La nueva globalidad justifica las ventajas comparativas entre los países más industrializados y contaminantes y los países pobres que revalorizan su capacidad para absorber los excesos de los países ricos y ofrecen los recursos genéticos 
y ecoturísticos de sus reservas de biodiversidad. La diferencia entre países centrales y periféricos ya no sólo se da por el pillaje y sobreexplotación visible de los recursos, sino que queda camuflada bajo las nuevas funciones asignadas a la naturaleza en las estrategias de apropiación de los bienes y servicios ambientales del planeta (Leff, et. al, 2002, pp. 478-486).

\section{CONCLUSIÓN}

Si se toma el ejemplo de la Amazonía y se proyecta a un planteamiento más general, podemos señalar que la compleja geopolítica de la globalización tiene en el tema medioambiental una nueva variable en donde a los espacios biogeográficos de América Latina, o por lo menos a un sector significativo de ellos, se le asignan un creciente valor natural-conservacionista estratégico, tanto en lo relativo a la conservación de la biodiversidad como frente al tema del calentamiento global. Se trata, por lo tanto, de una lógica que se hace extensible o todos los ecosistemas de América Latina, el Caribe y otros sectores del Sur del mundo "poco intervenidos", tales como los bosques fríos y templado lluviosos de Chile y Argentina, los distintos ecosistemas patagónicos, los glaciares y grandes reservas de agua dulce, los ecosistemas costeros y marinos, entre otros.

Vale decir, precisamente en la medida que se mantengan como ecosistemas naturales prístinos o poco intervenidos su valor aumenta en proporción directa a la agudización de la crisis ambiental. Y la tensión política mundial no sólo se expresa en la perspectiva tradicional de apropiarse de territorios de un Estado por parte de otro Estado, sino que ahora se trata de influir de manera determinante sobre el tipo de gestión que se hace sobre estos territorios que contienen un ecosistema clasificado como altamente vital o sensible para el equilibrio ecológico global, a fin de que se no se exploten industrialmente y se conserven prístinos y/o poco intervenidos.

Para nadie es un misterio que "un ambiente apropiado no puede obtenerse mediante los actos individuales de un país" (Thurow 1992, p. 261), y la crisis ambiental, con variables como el Cambio Climático, es un argumento que se utiliza reiteradamente sobre la necesidad de avanzar en un nuevo tipo de orden global, que vaya superando paulatinamente al tradicional sistema de Estados nacionales, con nuevas y cada vez más efectivas instituciones mundiales que puedan gestionar ambientalmente el planeta como un todo. De hecho, el expresidente chileno, Ricardo Lagos, actuando como enviado especial del Secretario General de la ONU a la Cumbre sobre Cambio Climático, realizada en Bali en 2007, señaló que si bien a nadie la gusta ceder soberanía, a raíz del Cambio Climático se van a tener que "modificar nuestros estilos de vida, nuestra forma de organizar las sociedades... Implica aceptar que los estados van a perder parte de su soberanía. Porque tiene que haber una soberanía global, porque es un problema global" (Luco, El Mercurio, Santiago, 2 diciembre de 2007).

Sin embargo, en la medida que no se logren acuerdos multilaterales efectivos y satisfactorios para todos los países, tanto desarrollados como en vías de desarrollo, y que las nuevas instituciones globales emergentes o por emerger sean capaces de dar plena garantía de justicia a todos los actores del sistema, esta tensión puede ser crecientemente compleja y determinante para la política mundial de la primera mitad 
del presente siglo XXI. La falta de acuerdos multilaterales no detiene la crisis ambiental, por el contrario, la agrava. $Y$, en este sentido, no se puede descartar que surjan medidas para mitigarla más o menos unilaterales (por difíciles que sea imaginarlas hoy día), sobre todo por parte de los Estados más poderosos y desarrollados, en la medida que la crisis se agrave y su preocupación aumente. Recuérdese que la Ministra de Defensa de Argentina, Nilda Garré, al término de la cumbre del UNASUR celebrada en agosto del 2009 en Bariloche, respecto del establecimiento de bases militares estadounidenses en Colombia, señaló que "la preocupación latente en la Argentina, que también se repite en otros presidentes, gira en torno de la hipótesis de que el desembarco norteamericano en la región pueda tener como propósito la observación y registro de los recursos naturales..." (Colonna, La Nación, Buenos Aires, 30 de agosto 2009, p. 2).

\section{BIBLIOGRAFÍA}

Anderson L.; Terry; Leal R. Donald, Ecología de Mercado. Madrid, Unión Editorial, 1993.

Bindé, Jérôme, "El porvenir de la Tierra ¿Qué futuro para la humanidad?". Política Exterior, núm. 123. mayo / junio 2008, pp. 103-112.

Campbell, Kurt M. (et.al), The Age of Consequences:Policy and National Security. Implications of global climate chang. Washington D.C., Center for Strategic \& International Studies and Center for a New American Security, 2007.

Christopher, Warren, "Diplomacia de los EE.UU. y el Medio Ambiente", en Boletín de Prensa del Servicio Informativo y Cultural de los Estados Unidos, Av. Andrés Bello 2800, Santiago-Chile, 15 de abril de 1996.

Caride, José Antonio; Meira, Pablo Ángel, Educación Ambiental y Desarrollo Humano. Barcelona, Ariel, 2001.

Ciemat, "Situación de la energía en el mundo, Europa y España". Gobierno de España, Ciemat, Febrero 2006 (www.energiasrenovables.ciemat.es/ especiales / energia/index.htm\#1).

Comisión de Desarrollo y Medio Ambiente de América Latina y el Caribe, Nuestra Propia Agenda; Banco Interamericano de Desarrollo, Programa de Naciones Unidas para el Desarrollo, 1991.

Díaz Peña, Luis Alberto; Jaramillo Gómez, Jaime, Tratado de Cooperación Amazónica. Trabajo de investigación presentado al Colegio Interamericano de Defensa como requisito para la obtención del diploma aprobatorio del curso superior de Defensa Continental. Washington DC, Colegio Interamericano de Defensa. Departamento de Estudios. Curso XXXV, 1996. 
Ehrlich, Paul E. y Ehrlich, Anne H., La Explosión Demográfica. El principal problema ecológico. Barcelona, Salvat, 1993.

Estenssoro Saavedra, Fernando. Medio Ambiente e Ideología. La discusión pública en Chile, 1992-2002. Santiago, Ariadna/USACH, 2009.

“El fracaso de la cumbre de La Haya sobre el Cambio Climático", Mapping Interactivo. Revista Internacional de Ciencias de la Tierra $\mathrm{N}^{\circ}$ 72, septiembre/octubre de 2001. ISSN: 1.131-9.100. http://www.mappinginteractivo.com/plantilla-ante. asp?id_articulo=63

Fregapani, Gelio, Amazónia. A Grande Cobija Internacional. Brasilia, Thesaurus, 2000.

Hannah, Lee; Carr, John L.; Lankerani, Ali, "Human disturbance and natural habitat: a biome level analysis of a global data set". Biodiversity and Conservation, Volume 4, Number 2 / marzo de 1995, pp. 128-155.

Homer-Dixon, Thomas, Carbon Shift. How the twin crises of oil depletion and Climate Change will define the future. Canadá, Random House, 2009.

Homer-Dixon, Thomas, The Upside of Down. Catastrophe, creativity, and the renewal of civilization. Canadá, Alfred A. Knopf / Random House, 2006.

International Energy Agency (IEA), Key World Energy Statistics 2006, (http://www. iea.org).

Intergovernmental. Panel on Climate Change, IPCC, Climate Change 2007. The physical science basis. Cambridge, Cambridge University, 2007.

Intergovernmental Panel on Climate Change, IPCC, Climate Change 2007. Synthesis Report. Sweden, IPCC, 2008.

Isbell, Paul, "Política energética de Obama después de un año". Economía Exterior No51 - Invierno 2010 (http:/ / www.politicaexterior.com).

Joxe, Alain (2003), El Imperio del Caos. Las repúblicas frente a la dominación estadounidense en la posguerra fría. Buenos Aires, Fondo de Cultura Económica.

Leff, Enrique (et.al), “Más Allá del Desarrollo Sostenible: La construcción de una racionalidad ambiental para la sustentabilidad. Una visión desde América Latina". En, Leff, Enrique; Ezcurra, Exequiel; Pisanty, Irene; Romero Lankao, Patricia (compiladores); La Transición Hacia el Desarrollo Sustentable. Perspectivas de América Latina y el Caribe. México D.F., SEMARNAT, INE, Universidad Autónoma Metropolitana, ONU, PNUMA, 2002, pp. 447-576. 
Llanos Mancilla, Hugo, Teoría y práctica del derecho internacional público. Tomo II. Santiago, Editorial Jurídica, 1980.

National Intelligence Council (NIC). Global Trends 2029: A Transformed World. Washington DC, US Government Printing Office, 2008 (www.dni.gov/nic/ NIC 2025 project.html).

Nogué Font, Joan; Vicente Rufí, Joan; Geopolitica, identidad y globalización. Barcelona, Ariel (2001).

Nye S., Joseph jr. (2003). La Paradoja del Poder Norteamericano. Santiago, Taurus.

Olabe, Antxón y González, Mikel, "Cambio Climático, una amenaza para la seguridad global”. Politica Exterior, núm. 124, Julio/ Agosto 2008, pp. 175- 185.

Palazuelos, Enrique (director), El petróleo y el gas en la geoestrategia mundial. Madrid, Akal, 2008.

Procopio, Argemiro, Quo Vadis, Amazonia? Buenos Aires, Nuevo Hacer, 2009.

Programa de Naciones Unidas para el Medio Ambiente (PNUMA). Perspectivas del Medio Ambiente Mundial. GEO 4. Medio ambiente para el desarrollo. Dinamarca, PNUMA, 2007.

Rodríguez U., Manuel Luis, "Seguridad y defensa en el siglo XXI - Elementos para un análisis geopolítico". En, GeopolíticaSur. Perspectivas y análisis geopolítico desde el sur del planeta, febrero 2010 (http: / / geopoliticasur.wordpress.com)

Ruiz-Eldredge, Alberto. "Nacionalismo y conflicto en América Latina". Nueva Sociedad, No 40 , enero-febrero, pp. 5-18.

Sánchez Joan -Eugeni (1992). Geografía Política. Madrid, Editorial Síntesis.

Schoijet, Mauricio, Límites del Crecimiento y Cambio Climático. México DF, Siglo XXI, 2008.

Sohr, Raúl, Chao, Petróleo. Santiago, Debate, 2009.

Taylor, J. Peter; Flint, Colin, Geografía Política. Economía-Mundo, Estado-Nación y Localidad. Madrid, Trama Editorial, 2002.

Thurow, Lester, La Guerra del Siglo XXI. Buenos Aires, Editorial Vergara, 1992.

Tickell, Oliver, Kyoto 2. Cómo gestionar el efecto invernadero global. Barcelona, Icaria, 2009. 
United States Joint Forces Command, The JOE 2010. The Joint Operating Environment. Suffolk, 2010.

Ward, Bárbara y Dubos, René, Una Sola Tierra. El cuidado y conservación de un pequeño planeta. México D.F., Fondo de Cultura Económica, 1984.

World Summit on Sustainable Development, "World Summit declares 'fault line' between rich and poor threatens prosperity, adopts broad measures to alleviate poverty, protect environment". Department of Public Information - News and Media Services Division - New York. ENV/DEV/J/35. 04 de septiembre 2002. www.un.org/events/wssd/summaries.

Working Group III of the Intergovernmental Panel on Climate Change (IPCC); Climate Change 2007.Mitigation of Climate Change. Summary for policymakers and Technical Summary. Intergovernmental Panel on Climate Change, 2007.

Zanelli, Jorge, "Energía nuclear: ¿riesgo u oportunidad?". Ponencia en Seminario Energía y Medio Ambiente. Una ecuación difícil para América Latina. Santiago, USACH, 15 de octubre de 2009.

\section{Fuentes de Prensa:}

Colonna, Lucas, “La ministra de Defensa se refirió al acuerdo alcanzado por la región en la cita de Bariloche". La Nación, Buenos Aires, 30 de agosto de 2009.

"Ecuador pedirá compensación por no explotar su petróleo". El Mercurio, Santiago, 11 de diciembre de 2008.

Luco Rojas, Nicolás, “Habrá que entregar parte de la soberanía”. El Mercurio, Santiago, 2 diciembre de 2007.

"Obama aprueba exploración petrolífera en el Atlántico Norte y Alaska". La Tercera, Santiago, 1 de abril de 2010.

"Obama hace frente al problema del Cambio Climático y la dependencia energética". El Mercurio, Santiago, martes 27 de enero de 2009 (http:/ / diario. elmercurio.cl).

Rojas, Pablo, "Cumbre Mundial de los Pueblos por el Cambio Climático". El Siglo, Santiago, 23 al 29 de abril de 2010.

Townsend, Mark y Harris, Paul, "Now the Pentagon tells Bush: Climate Change will destroy Us"; The Observer, February 22, 2004.

Vera, Franco y Luco, Nicolás, "Urge acuerdo por territorios del Ártico". El Mercurio, Santiago, 25 de julio de 2008.

Sauven, John, "La Amazonía se muere". La Nación; Santiago, 10 de junio de 2009.

"Brasil defiende soberanía sobre Amazonía ante EE.UU". La Nación, Santiago, 3 de marzo de 2009. 


\section{Otras páginas web}

www.ambientum.com

http: / / ecos.blogalia.com/historias/53937

www.ccccok.org/museum/dustbowl.html

http:/ / www.cinu.org.mx 